\title{
FEROMÔNIOS ASSOCIADOS AOS COLEÓPTEROS-PRAGA DE PRODUTOS ARMAZENADOS
}

\author{
Marcos Antônio Barbosa Moreira \\ Departamento de Zoologia, Universidade Federal do Paraná, CP 19030, 81531-990 Curitiba - PR \\ Paulo Henrique Gorgatti Zarbin* e Miryan Denise Araújo Coracini \\ Departamento de Química, Universidade Federal do Paraná, CP 19081, 81531-990 Curitiba - PR
}

Recebido em 13/4/04; aceito em 27/10/04; publicado na web em 17/2/05

\begin{abstract}
PHEROMONES ASSOCIATED TO COLEOPTERAN PESTS IN STORED PRODUCTS. One strategy to overcome risks of insecticide-based control in agriculture is to use semiochemicals. In the case of pheromones, these specific compounds can be applied in traps to detect and monitor the occurrence, abundance and distribution of insect pests. Reliable detection helps to time insecticide sprays, to decide the quantity of insecticide that will be used and the place where it will be applied. This manuscript aims to give an overview of the pheromones associated to coleopteran pests in stored products, and their utilization in integrated pest management.
\end{abstract}

Keywords: pheromones; stored products; IPM.

\section{INTRODUÇÃO}

A crescente preocupação em preservar o ecossistema tem exigido da comunidade científica a busca por novos métodos de controle de insetos-praga. O uso indiscriminado de agrotóxicos, além de causar danos ao meio ambiente e organismos não-alvos, tem causado problemas relacionados à resistência dos insetos aos princípios ativos destes produtos ${ }^{1}$. As perdas mundiais em produtos armazenados, devido ao ataque de insetos-praga na pós-colheita, são estimadas anualmente em $15 \%$ e custos elevados são envolvidos para a proteção destes produtos contra estas infestações ${ }^{2}$. No Brasil, as perdas chegam a atingir $10 \%$ de toda a produção, sendo os principais fatores associados ao ataque de insetos, fungos e ácaros nos armazéns, silos e depósitos agroindustriais durante o armazenamento (Figura 1$)^{3}$.

O estudo do comportamento dos insetos tem resultado no desenvolvimento de novos métodos que estão sendo usados em programas de manejo integrado de insetos-praga. O uso de semioquímicos, principalmente os feromônios, está se tornando uma importante ferramenta para a implementação de alternativas para o monitoramento e controle de insetos-praga de produtos armazenados $^{1}$.

O objetivo desta revisão é descrever a comunicação química dos principais coleópteros-praga associados aos produtos armazenados, enfatizando os feromônios identificados, e discutir viabilidades e perspectivas de aplicação destes compostos como forma alternativa para o controle das pragas. a)

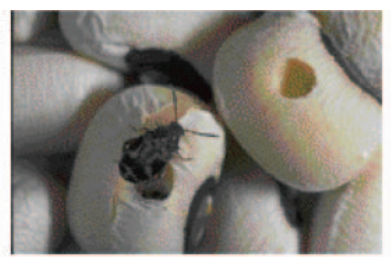

b)

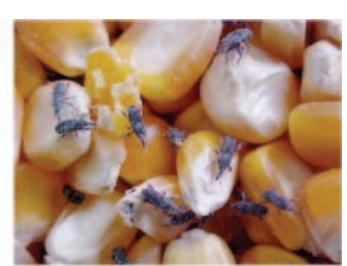

Figura 1. Insetos causando danos em produtos armazenados. (a) Acanthoscelides obtectus em feijão; (b) - Sitophylus zeamais em milho

\section{A COMUNICAÇÃO QUÍMICA E AS ESTRATÉGIAS REPRODUTIVAS}

Parte integrante do comportamento animal é a comunicação, definida como um processo que envolve a transmissão de sinais entre organismos. Em algumas situações confere vantagens apenas para o organismo emissor e seu grupo; em outras, apenas para os organismos receptores ou para ambos ${ }^{4}$.

Os insetos são os seres vivos que mais utilizam os odores para desempenhar suas funções vitais (localização de presas, defesa e agressividade, seleção de plantas hospedeiras, escolha dos locais para oviposição, corte e acasalamento) $)^{5}$. Essa comunicação pode ser efetuada entre organismos da mesma espécie (feromônios substâncias secretadas por um indivíduo e recebidas por um segundo, provocando uma reação específica ou um processo de desenvolvimento fisiológico específico ${ }^{5}$ ) ou entre espécies diferentes (cairomônios, alomônios). Os feromônios sexuais e de agregação são os mais freqüentes em pragas dos produtos armazenados e têm sido mais estudados, pois se enquadram melhor em estratégias de controle de pragas ${ }^{6}$.

As pragas dos produtos armazenados apresentam, basicamente, dois tipos de comunicação e estratégia reprodutiva ${ }^{7}$. A primeira refere-se aos insetos que possuem vida curta (menor que um mês) e não necessitam de um "sítio" de alimentação para o processo de reprodução. Os insetos de vida curta, como Dermestídeos, Bruchídeos e Anobiídeos, utilizam-se exclusivamente dos feromônios sexuais produzidos pelas fêmeas, que atraem os indivíduos co-específicos para o acasalamento. A segunda refere-se àqueles insetos que possuem vida longa (maior que um mês) e que dependem essencialmente dos alimentos para se reproduzirem. Nos insetos que utilizam esta estratégia, como os Bostrichídeos, Cucujídeos, Curculionídeos, Silvanídeos, Tenebrionídeos, Bruchídeos e em várias espécies de Cerambicídeos, os machos da espécie produzem feromônios de agregação, cuja função é atrair indivíduos de ambos os sexos para as fontes de alimento onde, então, ocorrerá o acasalamento ${ }^{7,8}$.

O entendimento da vantagem evolutiva dos machos que produzem feromônios é bastante complexo, uma vez que não há atração exclusiva das fêmeas para o acasalamento, mas também de com-

*e-mail: pzarbin@quimica.ufpr.br 
petidores co-específicos. Uma das explicações é que a função primária original da especificidade da produção de feromônio pelos machos é, comprovadamente, potencializar a atração e efetuar o acasalamento 9 . Neste contexto, a função primária para a produção de feromônio de agregação dos insetos machos é atrair outros machos e fêmeas para vencer as defesas do hospedeiro ou microclimas inadequados ${ }^{10}$. Acasalamentos por meio da mediação de feromônios de agregação têm uma importância secundária, desde que adultos das espécies que utilizam este feromônio possuam vida relativamente longa. Este sistema é análogo à estratégia que usam alguns Escolítideos. Nestes espécimes, durante a primeira fase em que os insetos adultos se agregam em uma planta hospedeira, o feromônio emitido pelos insetos pioneiros recruta insetos suficientes para vencer a resistência e as defesas do hospedeiro ${ }^{11}$. Somente um número específico de indivíduos, independentemente do sexo, chega no hospedeiro em curto tempo para realizar as tarefas primárias. Assim, os machos devem primeiro localizar a fonte de alimento antes de poder atrair os seus co-específicos ${ }^{11}$.

A maioria dos coleópteros, provavelmente, utiliza os feromônios de agregação visando atenuar a resistência do hospedeiro e deixar este habitat mais adequado ${ }^{12}$. Em ambiente de armazenagem, onde sementes e outros produtos pós-colheita são mantidos sob condições de baixas temperaturas e umidade relativa do ar, o feromônio de agregação pode funcionar para reunir um grande número de indivíduos com o propósito de alterar as condições microclimáticas desse local ${ }^{11}$, as quais são parcialmente provocadas pelos metabolismos dos produtos e dos insetos-praga. Sob estas condições ambientais específicas poderia haver, por parte de determinadas espécies, uma necessidade pré-adaptativa dos feromônios de agregação visando possibilidades de obtenção de êxito nas infestações de insetos adultos, na alimentação e sobrevivência em produtos armazenados ${ }^{10}$.

Os feromônios de agregação também podem funcionar na regulação de densidade populacional ${ }^{13}$. Altas densidades populacionais causam redução na fecundidade, debilitam a oviposição e aumentam a mortalidade ${ }^{14}$. Entretanto, coleópteros que apresentam altas densidades populacionais são menos atraídos pelo feromônio de agregação ou, sob determinadas condições, podem até serem repelidos pela ação do mesmo ${ }^{14}$. Em certas espécies, como Oryzaephilus surinamensis (Coleoptera: Cucujidae), os insetos, quando mantidos sob condições de baixas densidades populacionais, chegam a produzir duas vezes mais feromônio que quando mantidos sob altas densidades ${ }^{14}$.

\section{COLEÓPTEROS-PRAGA E SEUS FEROMÔNIOS}

Praticamente todos os feromônios de insetos em produtos armazenados de interesse econômico já foram identificados, sendo que a primeira espécie que teve seu feromônio sexual identificado foi o besouro Attagenus unicolor (Coleoptera: Dermestidae). Abaixo estão descritas as principais espécies e seus feromônios associados.

\section{Lasioderma serricorne (Coleoptera: Anobiidae)}

Ocorre principalmente em tabaco armazenado, mas também em fábricas de processamento de alimentos e em alimentos de animais em "pet-shops". As fêmeas são as responsáveis pela liberação de um feromônio sexual e somente os machos são atraídos. O principal componente feromonal foi identificado por Chuman e colaboradore ${ }^{15}$ como sendo a $(4 S, 6 S, 7 S)$-7-hidroxi-4,6-dimetil-3nonanona (1), serricornina. Mori e colaboradores ${ }^{16}$ definiram a configuração absoluta do isômero responsável pela atratividade. Esta espécie produz ainda a $\left(2 S, 3 R, 1^{\prime} R\right)$-2,3-diidro-2-etil-3,5-
dimetil-6-(1'-metil-2-oxobutil)-4H-piran-4-ona (2), serricorona, e a $\left(2 S, 3 R, 1^{\prime} S, 2\right.$ ' $\left.S\right)$-2,3-diidro-2-etil-3,5-dimetil-6-(1'-metil-2'hidroxibutil)-4H-piran-4-ona (3), serricorola, os quais elicitam nos insetos machos comportamentos de agregação e de acasalamento ${ }^{15}$. A relação entre $\mathbf{1}, \mathbf{2}$ e 3 foi determinada como sendo $4,4: 1,7: 1^{17}$.<smiles>CCC(=O)C(C)CCC(O)CC</smiles>

1<smiles>CCC(=O)C(C)C1=C(C)C(=O)[C@H](C)[C@H](CC)O1</smiles>

2<smiles>CC[C@H](O)C(C)C1=C(C)C(=O)[C@H](C)[C@@H](CC)O1</smiles>

3

\section{Stegobium paniceum (Coleoptera: Anobiidae)}

Ocorre em moinhos de grãos, em locais de armazenamento de alimentos e de especiarias (temperos). As fêmeas são as responsáveis pela liberação do feromônio sexual e somente os machos da espécie são atraídos. As fêmeas tornam-se atrativas para os machos com 4 a 5 dias de idade, e o período máximo de atratividade verifica-se com uma semana após a emergência ${ }^{18}$. A identificação deste feromônio foi realizada em 1978 por Kuwahara e colaboradores ${ }^{19}$ como sendo o $\left(2 S, 3 R, 1^{\prime} R\right)$-2,3-diidro-2,3,5-trimetil-6-(1'metil-2-oxobutil)-4H-piran-4-ona (4), estegobinona, cuja configuração absoluta foi estabelecida através da síntese realizada por Hoffmann e colaboradores ${ }^{20}$. Posteriormente, Kodama e colaboradores $^{21}$ identificaram um segundo componente feromonal, um álcool análogo identificado como ( $2 S, 3 R, 1$ ' $S, 2$ ' $S$ )-2,3-diidro-2,3,5trimetil-6-(2'-hidroxi-1'-metilbutil)-4H-piran-4-ona (5), estegobiol, e constatou-se que este composto opera como atraente sexual à curta distância, enquanto 4 atua à longa distância ${ }^{21}$.

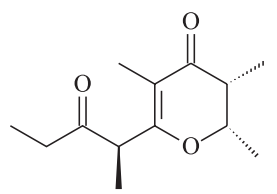

4<smiles>CC[C@H](O)C(C)C1=C(C)C(=O)[C@H](C)[C@H](C)O1</smiles>

5

\section{Rhyzopertha dominica (Coleoptera: Bostrichidae)}

Principal praga de pós-colheita do trigo no Brasil, mas também se alimenta de cevada, triticale, arroz e aveia. $\mathrm{O}$ feromônio de agregação para ambos os sexos desta espécie é composto pelos componentes (E)-2-metil-2-pentenoato de 1-metilbutila (6), dominicalure 1, e (E)-2,4-dimetil-2-pentenoato de 1-metilbutila (7), dominicalure 2, os quais são efetivos individualmente ou em várias combinações ${ }^{22}$. A produção e a concentração dos componentes feromonais 6 e 7 variam continuamente em um período de um mês.<smiles>CCC=C(C)C(=O)OCCCC</smiles>

6<smiles>CCCCOC(=O)/C(C)=C/C(C)C</smiles> 
Não há influência da idade nos níveis de produção de feromônio, ou do número de cópulas já realizadas pelo inseto ${ }^{23}$. Os isômeros naturais $(S)$ mostraram-se duas vezes mais ativos em testes de campo que os respectivos enantiômeros ${ }^{24}$.

\section{Acanthoscelides obtectus (Coleoptera: Bruchidae)}

É uma das principais pragas do feijão. Os machos desta espécie produzem um feromônio que é atrativo apenas para fêmeas virgens, sendo que estas, aparentemente, não produzem feromônio sexual. Após a cópula, as fêmeas não são atraídas para outros machos durante o resto de suas vidas ${ }^{25}$. A estrutura química deste feromônio foi definida como sendo o $(R, E)$-2,4,5-tetradecatrienoato de metila $(\mathbf{8})^{26}$.

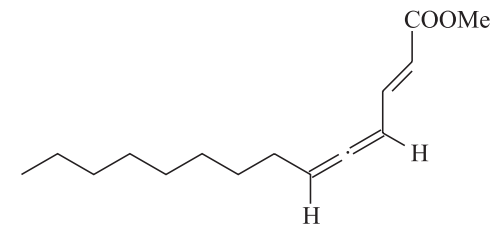

8

\section{Zabrotes subfasciatus (Coleoptera: Bruchidae)}

É uma das únicas espécies-praga de produtos armazenados cujo feromônio ainda não foi identificado. Entretanto, vários fatores que envolvem a regulação da produção de um eventual feromônio têm sido descritos na literatura ${ }^{27}$. A produção e/ou a liberação do feromônio está aparentemente correlacionada com a maturidade sexual dos adultos. Deterrentes de oviposição têm sido relatados em várias espécies de bruchídeos, denominados feromônios marcadores de hospedeiros (HMP) ${ }^{28}$, os quais impedem que ocorra oviposição de uma outra espécie nas sementes do feijão.

\section{Cryptolestes spp. (Coleoptera: Cucujidae)}

Praga que ocorre em grãos quebrados, restos de grãos e farinhas em armazéns. Algumas espécies do gênero já tiveram seus feromônios identificados. Para $C$. ferrugineus, o feromônio de agregação foi identificado como sendo uma mistura de $(4 E, 8 E)-4,8$ dimetil-4,8-decadien-10-olida (9), ferrulactona I, e (3Z,11S)-3dodecen-11-olida (10), ferrulactona II, as quais atuam de maneira sinergística ${ }^{29}$. As fêmeas são mais sensíveis a estes feromônios que os machos. Em condições naturais, a proporção observada entre os dois componentes é de 1,6:1, entretanto, a proporção de 9:1 é que melhor promove uma resposta comportamental ${ }^{30}$. Millar e colaboradores $^{31}$ identificaram três outros componentes do feromônio de agregação de $C$. pusillus. A (Z)-3-dodecen-12-olida (11) é o componente majoritário, apresentando atividade quando testado isoladamente. A $(5 Z, 13 S)$-5-tetradecen-13-olida (12) não possui atividade isoladamente, mas apresentou sinergismo considerável com a lactona 11. A (3Z,6Z)-3,6-dodecadien-12-olida (13) mostrou-se ativa em altas concentrações, mas não influenciou significativamente na atividade da mistura de $(S)-\mathbf{1 0}$ e $\mathbf{1 1}$.

\section{Oryzaephylus spp. (Coleoptera: Cucujidae)}

Este inseto também ocorre em todos os tipos de grãos quebrados e restos de grãos. As estruturas químicas atribuídas aos feromônios dessas espécies são bastante próximas àquelas mencionadas anteriormente, por pertencerem a mesma família. O

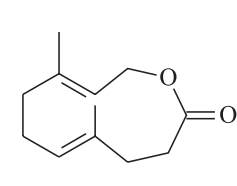

9

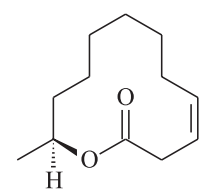

$(S)-10$

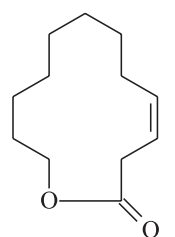

11

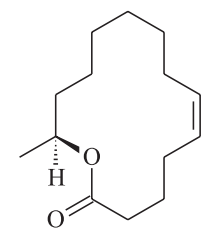

12

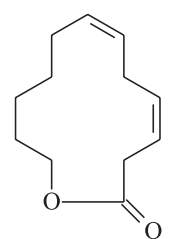

13 feromônio de agregação de $O$. mercator é composto por uma mistura $(1: 1,25)$ das macrolactonas $(R)-10$ e $(3 Z, 6 Z, 11 R)-3,6$ dodecadien-11-olida (14) ${ }^{32,33}$. Ambos os sexos desta espécie são atraídos para cada composto individualmente e, também, para uma mistura (1:1). Os insetos machos possuem uma menor resposta na atração para o feromônio $(R) \mathbf{1 0}$, enquanto que para as fêmeas uma menor atividade é observada para $14^{33}$. Na espécie $O$. surinamensis, uma mistura (1:3:1) das macrolactonas $\mathbf{1 3}, 14$ e $(5 Z, 8 Z, 13 R)-5,8$ tetradecadien-13-olida (15) foi atribuída como feromônio de agregação, sendo necessária a presença dos três componentes para uma efetiva atratividade ${ }^{33}$.

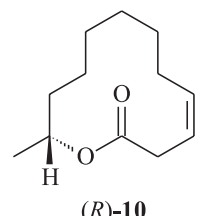

(R)-10

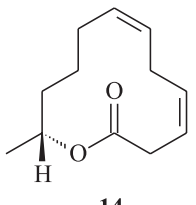

14

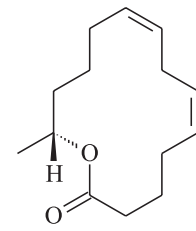

15

\section{Sitophylus spp. (Coleoptera: Curculionidae)}

Também chamados de gorgulhos dos cereais, pois atacam milho, trigo, arroz, cevada, etc. Os feromônios de agregação ocorrem em $S$. oryzae, $S$. zeamais e $S$. granarius. Phillips e colaboradores ${ }^{34}$ identificaram o $(4 S, 5 R)$-5-hidroxi-4-metil-3-heptanona (16), sitophilure, como sendo o feromônio de agregação de $S$. oryzae e $S$. zeamais. A estrutura química do feromônio de $S$. granarium foi também identificada por Phillips e colaboradores ${ }^{35}$ como sendo o $^{\circ}$ (2S,3R)-2-metil-3-hidroxipentanoato de 1-etilpropila (17), sitophilato. Para todas estas espécies, a presença dos feromônios em altas concentrações reduz as atrações ou até causa efeitos de repelência ${ }^{36,37}$.<smiles>CCC(=O)C(C)[C@H](O)CC</smiles><smiles>CCC(CC)OC(=O)[C@H](C)[C@H](O)CC</smiles>

\section{Anthrenus spp. (Coleoptera: Dermestidae)}

Ocorrem em lã e seus sub-produtos armazenados e em objetos de museus. As fêmeas de A. flavipes apresentam o comportamento de chamamento próximo do início da fotofase, correlacionado com a liberação de um feromônio sexual, o ácido 3-decenóico $(\mathbf{1 8})^{38}$. As fêmeas de A. verbasci também liberam feromônio sexual que atrai machos e estimula sua resposta sexual. Para esta espécie, o 
feromônio foi identificado como uma mistura 85:15 dos isômeros $(Z)$ e $(E)$ do ácido 5-undecenóico (19) ${ }^{39}$.

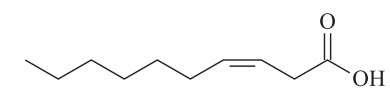

18

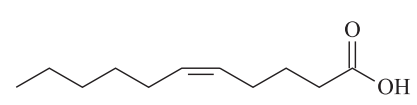

19

\section{Attagenus spp. (Coleoptera: Dermestidae)}

Ocorrem preferencialmente em locais onde há processamento de alimentos, mas também foram descritos em objetos de museu, em sêda armazenada, e até mesmo alimentando-se em pupas do bicho da sêda. Fêmeas maduras de várias espécies deste gênero demonstraram comportamento de chamamento diurno, o qual está associado com a liberação de um feromônio sexual ${ }^{7,40,41}$. Em $A$. elongatulus e A. rufipennis, a emissão de feromônio pelas fêmeas e a resposta do macho ocorrem durante as primeiras horas da fotofase, enquanto que nas espécies A. unicolor e A. bicolor o comportamento de chamamento é efetuado durante a metade do período da fotofase. Esses insetos utilizam-se, comumente, do ácido 3,5-tetradecadienóico como feromônio sexual. Fêmeas de $A$. megatoma emitem o ácido (3E,5Z) megatomóico (20), enquanto que A. elongatulus produz o isômero (3Z,5Z)- 21.

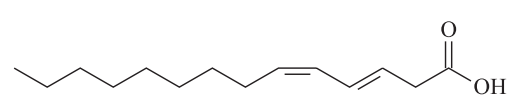

20

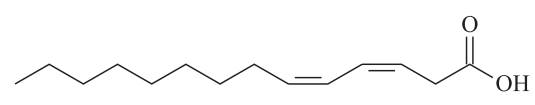

21

\section{Trogoderma spp. (Coleoptera: Dermestidae)}

Ocorrem em trigo armazenado, em fábricas de processamento de alimentos, em sêda armazenada e até mesmo alimentando-se em pupas do bicho da sêda. Diversas espécies de Trogoderma produzem o (Z)- ou (E)-14-metil-8-hexadecenal (22), trogodermal, como feromônio sexual ${ }^{42,43}$. O isômero $(R)$-22 tem sido descrito como o enantiômero bioativo ${ }^{44}$, apesar de Rossi e colaboradores ${ }^{45}$ mencionarem que a atividade está associada ao isômero $(S)$. Foram relatadas respostas interespecíficas para extratos de fêmeas de várias espécies de Trogoderma, e atração cruzada de machos para fêmeas não co-específicos ${ }^{46}$. A atração cruzada nestas espécies foi explicada pela ocorrência de substâncias precursoras do feromônio, o (Z)-14-metil-8-hexadecenol (23) e (Z)-14-metil-8-hexadecenoato de metila (24) em extratos de fêmeas de T. inclusum, T. variabili e T. granarium $^{47}$.

\section{Tenebrio molitor (Coleoptera: Tenebrionidae)}

Ocorre em fábricas de processamento de alimentos e em locais de armazenamento de farinha. Vários feromônios podem estar envolvidos na mediação e no comportamento de cópula nesta espé$\mathrm{cie}^{48}$. Feromônio produzido pelas fêmeas atrai machos; feromônio produzido pelos machos atrai fêmeas; um ou mais feromônios in-

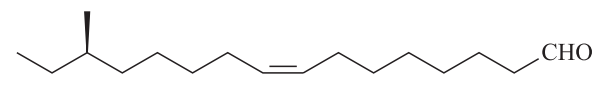

(Z)-22

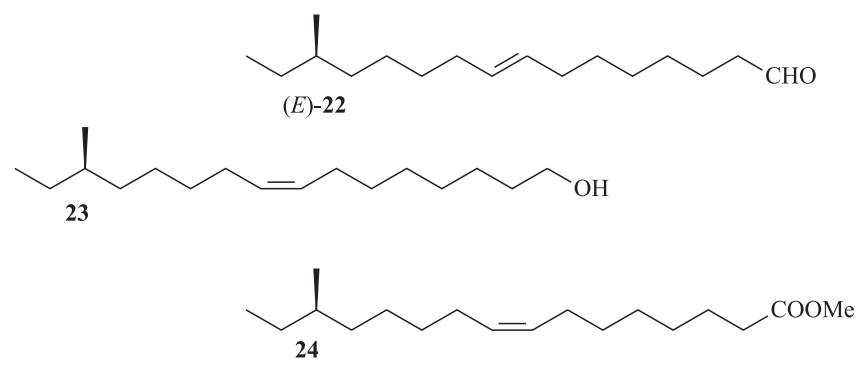

duzem a cópula e produção de feromônio anti-afrodisíaco produzido pelos machos ${ }^{49}$. O feromônio sexual produzido pelas fêmeas foi identificado por Tanaka e colaboradore ${ }^{50}$ como sendo o $(R)$-4-metil1-nonanol (25) $)^{51}$. A produção aumenta com a maturação sexual das fêmeas, atingindo o pico máximo em 4-5 dias após a emergência. A resposta comportamental dos machos frente ao feromônio sexual das fêmeas é semelhante, aumenta com a idade e maturidade, alcançando o pico máximo em 5 dias após a eclosão ${ }^{51}$. Sob condições naturais, o feromônio das fêmeas pode facilitar a cópula, desde que tenham sido efetuados contatos físicos. $\mathrm{O}$ isômero $(R)$ sintético mostrou atividade semelhante ao produto natural, enquanto seu enantiômero $(S)$ não apresentou nenhum efeito sinergístico ou $\operatorname{antagônico~}^{50}$.<smiles>CCCCCC(C)CCCO</smiles>

\section{Tribolium spp. (Coleoptera: Tenebrionidae)}

Estes insetos dependem do ataque de outras pragas para se instalarem nos grãos armazenados, alimentando-se de várias espécies como sorgo, trigo, milho. O macho é o responsável pela liberação de feromônio de agregação em várias espécies, como T. confusum, T. castaneum, T. brevicornes, T. destructor, T. audax e T. freemani ${ }^{52,53}$. O principal componente associado a este gênero é o $(4 R, 8 R)-4,8$ dimetildecanal (26), tribolure ${ }^{54}$, cuja estereoquímica foi definida por Mori e colaboradores ${ }^{55}$. Posteriormente, bioensaios realizados por Suzuki e colaboradores ${ }^{56}$ revelaram que uma mistura 8:2 dos isômeros $(4 R, 8 R)-\mathbf{2 6}$ e $(4 R, 8 S)-\mathbf{2 7}$ foi 10 vezes mais ativa que o isômero natural isolado.<smiles>CCC(C)CCCC(C)CCC=O</smiles><smiles>CCCCCCC(C)CCC=O</smiles>

\section{MÉTODOS DE CONTROLE DE PRAGAS DE PRODUTOS ARMAZENADOS}

Os métodos de controle utilizados no Brasil são os físicos e os químicos. Os métodos físicos envolvem a manipulação dos fatores físicos para reduzir a população da praga a um nível tolerável ou eliminá-la ${ }^{57}$. Controle da temperatura, da umidade relativa e da composição da atmosfera $\left(\mathrm{CO}_{2}, \mathrm{O}_{2}, \mathrm{~N}_{2}\right)$, uso de pós-inertes, remoção física de insetos, radiação ionizante, luz e som podem ser empregados, isoladamente ou combinados, para se obter o controle de pragas $^{3}$. O uso de inseticidas é um dos métodos de controle mais empregados na atualidade. O controle químico pode ser aplicado 
de forma preventiva ou curativa ${ }^{3}$. Porém, há o problema do surgimento de resistência das pragas aos inseticidas.

\section{Aplicação de feromônios no controle de pragas de produtos armazenados}

Atualmente há no mercado produtos (armadilhas) contendo feromônios para serem utilizados no monitoramento de algumas espécies de pragas de produtos armazenados. É possível encontrar, por exemplo, produtos para as espécies C. ferrugineus, C. pusillus, $O$. mercator, O. surinamensis, $R$. dominica, S. oryzae, S. zeamais, S. granarius, T. castaneum, T. confusum, T. granarium e T. variable ${ }^{58}$. Contudo, no mercado brasileiro, há apenas produtos para as espécies $T$. castaneum, recomendados para serem empregados em grãos armazenados e seus subprodutos, e L. serricorne, para ser empregado em fumo armazenado ${ }^{59}$.

Estudos estão sendo conduzidos com o objetivo de desenvolver novos produtos para serem utilizados no controle de coleópterospragas, não apenas no seu monitoramento, mas também pelo método de confusão sexual, como já ocorre para lepidópteros-praga de frutas de clima temperado (maçã, pêssego, pêra e uva) ${ }^{60,61}$. Entretanto, isso já é algo mais difícil de ser aplicado aos coleópteros, pois o sistema de comunicação entre os indivíduos da mesma espécie pode envolver a interação entre vários fatores, não apenas o uso de feromônio sexual, como é o caso dos lepidópteros.

\section{VANTAGENS E DESVANTAGENS DO USO DE FEROMÔNIOS NO CONTROLE DE INSETOS}

Há vários benefícios quanto à utilização de feromônios para monitoramento e controle de insetos-praga. A principal vantagem do uso de feromônios sintéticos é que eles são espécie-específicos e atóxicos, ou seja, só vão atuar sobre as espécies-alvo. Quando utilizados no monitoramento de insetos, é necessário apenas uma pequena quantidade do produto, que é acondicionada em armadilhas. As armadilhas contendo feromônio auxiliam na previsão das épocas de adoção das metodologias para o controle curativo, aumentando a precisão do uso de inseticidas, na verificação da eficiência do tratamento químico convencional, e na detecção dos espécimes resistentes aos principais ingredientes ativos desses produtos. A utilização de feromônios sintéticos é um método de controle que pode ser incorporado às técnicas do manejo integrado de pragas (MIP).

Entretanto, como todo método de controle de pragas, o uso do feromônio apresenta algumas desvantagens. $\mathrm{O}$ custo das técnicas de controle de insetos através do uso de feromônios sintéticos, principalmente em estágios iniciais de instalação, é geralmente mais alto que as aplicações de inseticidas convencionais. Porém, a longo prazo este custo diminui. É preciso desenvolver estudos básicos sobre o inseto-praga e as interações com o ambiente para o método ser eficiente. Há dificuldades em relacionar o número de insetos capturados com os limiares de dano econômico das pragas. O processo de obtenção dos compostos feromonais com alta pureza estereoquímica requer, em alguns casos, metodologias sintéticas bastante complexas. E também, algumas vezes, os compostos isolados e identificados no laboratório não promovem uma resposta satisfatória no campo.

\section{CONSIDERAÇÕES FINAIS}

Apesar dos avanços bastante significativos na utilização dos feromônios para monitoramento e controle de insetos-praga, a sua aplicação ainda não foi suficiente para gerar tecnologias capazes de viabilizarem seu emprego em larga escala comercial, bem como ao pronto atendimento das demandas no controle de pragas. A prova inconteste desta afirmação é o aumento de novos produtos e avanços tecnológicos na área dos inseticidas convencionais que, apesar de toda a problemática ambiental envolvida, ainda continuam sendo a principal alternativa no controle de insetos-praga.

A escassez da divulgação e transferência de tecnologia destes produtos, a falta do engajamento da sociedade civil, a pouca conscientização dos produtores em geral e a falta de uma política pública visando fortalecer e ampliar o uso de feromônios na agricultura podem constituir os principais gargalos que inviabilizam os investimentos do setor privado, a aplicação em escala comercial e acessibilidade a esses produtos pelos usuários potenciais. Seria de fundamental importância a consolidação no Brasil de um grupo gestor em Ecologia Química, visando aglutinar esforços, angariar investimentos, efetuar prospecção de demandas tecnológicas a fim de reduzir custos com as pesquisas e sobreposição destas, e elaborar políticas públicas que trariam avanços significativos no uso destas substâncias.

\section{AGRADECIMENTOS}

Os autores agradecem à Fundação Araucária do Paraná, CNPq, CAPES, IFS(Suécia), OPCW(Holanda) e Embrapa/Prodetab pelos auxílios financeiros e bolsas concedidas.

\section{REFERÊNCIAS}

1. Ferreira, J. T. B.; Zarbin, P.H.G.; Química Nova na Escola 1998, nº 7, 3.

2. Reichmuth, C.; Schöller, M.; Ulrichs, C.; Gelsenkirchen 1997, 119.

3. Lorini, I.; Manual Técnico para o Manejo Integrado de Pragas de Grãos Armazenados, Embrapa Trigo, 2001.

4. Nascimento, R. R.; Sant'Ana, A. E. G. Em Feromônios de insetos: Biologia, Química e Emprego no Manejo de Pragas; Vilela, E. F.; Della Lúcia, T. M. C., eds.; 2 ${ }^{\mathrm{a}}$ ed., Holos Editora: Ribeirão Preto, 2001, p. 65-71.

5. Vilela, E. F.; Della Lucia, T. M. C. Em Feromônios de Insetos: Biologia, Química e Emprego no Manejo de Pragas; Vilela, E.F. e Della Lúcia, T.M.C., eds.; 1 ${ }^{\mathrm{a}}$ ed, Holos Editora: Ribeirão Preto, 1987, p. 155.

6. Nordlund, D. A.; Lewis, W. J.; J. Chem. Ecol. 1996, 2, 211.

7. Burkholder, W. E.; J. Georgia Entomol. Soc. 1982, 17, 1.

8. Boughton, A.; Fadamiro, H. Y.; J. Stored Prod. Res. 1996, 32, 13.

9. Landolt, P.J.; Am. Entomol. 1997, 43, 12.

10. Raffa, K. F.; Phillips, T. W.; Salon, S. M. Em Beetle. Pathogen Interactions in Conifer Forests; Schowater, T. D.; Phillips, G. M., eds; Acad. Press, 1993, p. 103.

11. Borden, J. H.; Dolinsk, M. G.; Chong, L.; Verigin, V.; Pierce, H. D. Jr.; Oehlschlager, A. C.; Can. Entomol. 1979, 111, 681.

12. Levinson, H. Z.; Levinson, A. R.; Anz. Schädlingskd. PFL 1994, 67, 47.

13. Mignon, J.; Haubruge, E.; Lienard, V.; Gaspar, C.; Lognay, G.; Entomol. Exp. Appl. 1996, 80, 555.

14. Pierce, A. M.; Borden J. H.; Oehlschlager, A. C.; J. Chem. Ecol. 1990, 16, 595.

15. Chuman, T.; Mochizuki, K.; Mori, M.; Kohno, M.; Kato, K.; Noguchi, M.; J. Chem. Ecol. 1985, 11, 417.

16. Mori, M.; Chuman, T.; Kohno, M.; Kato, K.; Noguchi, M.; Nomi, H.; Mori, K.; Tetrahedron Lett. 1982, 23, 667.

17. Imai, T.; Kodama, H.; Chuman, T.; Masahiro, K.J.; J. Chem. Ecol. 1990, $16,1237$.

18. Barratt, B. I. P.; Bull. Entomol. Res. 1974, 64, 621

19. Kuwahara, Y.; Fukami, H.; Howard, R.; Ishii, S.; Matsumura, F.; Burkholder, W.E.; Tetrahedron 1978, 34, 1769.

20. Hoffmann, R. W.; Ladner, W.; Steinbach, K.; Massa, W.; Schmidt, R.; Snatzke, G.; Chem. Ber. 1981, 114, 2786.

21. Kodama, H.; Ono, M.; Kohno, M.; Ohnishi, A.; J. Chem. Ecol. 1987, 13, 1871.

22. Khorramshahi, A, ; Burkholder, W. E.; J. Chem. Soc. 1981, 7, 33.

23. Dowdy, A. K.; Howard, R. W.; Seitz, L. M.; McGraughei, W. H.; Environ. Entomol. 1993, 22, 965

24. Willians, H. J.; Silverstein, R. M.; Burkholder, W. E.; Khorramshahi, A.; J. Chem. Ecol. 1981, 7, 759 . 
25. Halstead, D. G. H.; J. Stored Prod. Res. 1973, 9, 109.

26. Horler, D. F.; J. Chem. Soc. 1970, 6, 859.

27. Plarre, R.; Vanderwel, D. C. Em Pheromones of Non-lepidopteran Insects Associated with Agricultural Plants; Hardie, J.; Minks, A. K., eds.; CABI International: Oxford,1999, cap. 7.

28. Mbata, G. N.; Ramaswamy, S. B.; J. Stored Prod. Res. 1995, 31, 157.

29. Wong, J. H.; Verigin, V.; Oehlschlager, A. C.; Pierce, H. D. Jr.; J. Chem. Ecol. 1983, 9, 451 .

30. Clambers, J.; Morgan, C. P.; While P. R.; Mori, K.; Finnegan, D. E.; Pinniger, D. B.; J. Chem. Ecol. 1990, 16, 3353.

31. Millar, J. G.; Pierce, H. D. Jr.; Pierce, A. M.; Oehlschlager, A. C.; Border, J. H.; Barak, A. V.; J. Chem. Ecol. 1985, 11, 1053.

32. Oehlschlanger, A. C.; Pierce, A. M.; Pierce, H. D. Jr.; Borden, J. H.; J. Chem. Ecol. 1988, 13, 1543.

33. Pierce A. M.; Pierce, H. D. Jr.; Millar, J. G.; Borden, J. H.; Oehlschlanger A. C.; Proceedings of the International Working Conference on Stored Product Entomology, Manhattan, E.U.A., 1984.

34. Phillips, J. K.; Walgenbach, C. A.; Klein, J. A.; Burkholder, W. E.; Schmuff, N. R.; Fales, H. M.; J. Chem. Ecol. 1985, 11, 1263.

35. Phillips, J. K.; Miller, S. P. F.; Andersen, J. F.; Fales, H. M.; Burkholder, W. E.; Tetrahedron Lett. 1987, 28, 6145.

36. Levinson, H. Z.; Levinson, A.; Ren, Z.; Mori, K.; J. Appl. Entomol. 1990, $110,203$.

37. Jiang, X. L.; Entomol. Knowl. 1995, 32, 23.

38. Ma, M.; Burkholder, W. E.; Ann. Entomol. Soc. Am. 1978, 71, 129.

39. Kuwahara, Y; Nakamura.; J. Appl. Zool. 1985, 20, 354.

40. Silverstein, R. M.; Rodin, J. O.; Burkholder, W. E.; Gorman, J. E.; Science 1967, 157, 85 .

41. Barac, A. V.; Burkholder, W. E.; J. Chem. Ecol. 1978, 4, 451.

42. Cross, H. J.; Byler, R. C.; Cassidy, R. S.; Silverstein, R. M.; Greenblatt, R. E.; Burkholder, W. E.; Levinson, A. R.; Levinson, H. Z.; J. Chem. Ecol. 1976, 2, 457 .
43. Greenblatt, R. E.; Burkholder, W. E.; Cross, J. C.; Byler, C.; Silverstein, R. M.; J. Chem. Ecol. 1976, 2, 285.

44. Levinson, H. Z.; Mori, K.; Naturwiss. 1980, 67, 148.

45. Rossi, R.; Niccoli, A.; Naturwiss. 1978, 65, 259.

46. Vick, K. W.; Burkholder, W. E.; Gorman, J. E.; Ann. Entomol. Soc. Am. 1970, 66, 667.

47. Greenblatt, R. E.; Burkholder, W. E.; Cross, J. C.; Cassidy, R. F.; Silverstein, R. M.; Levinson, A. R.; Levinson, H. D.; J. Chem. Ecol. 1977, 3, 337.

48. August, C. J.; J. Insect Physiol. 1971, 17, 739.

49. Happ, G. M.; Nature 1969, 222,180.

50. Tanaka, Y.; Honda, H.; Ohsawa, K.; Yamamoto, I.; J. Pest. Sci. 1989, 14, 197.

51. Tanaka, Y.; Honda, H.; Ohsawa, K.; Yamamoto, I.; J. Pest. Sci. 1986, 11, 49.

52. Faustine, D. L.; Giese, W. L.; Phillips, J. K.; Burkholder, W. E.; J. Chem. Ecol. 1982, 8, 679

53. Susuki, T.; Nakakita, H.; Kuwahara, Y.; Appl. Entomol. Zool. 1987, 22, 340.

54. Susuki, T.; Sugarara, R.; Appl. Entomol. Zool. 1979, 14, 228.

55. Mori, K.; Kuwahara, S.; Ueda, H.; Tetrahedron 1983, 39, 2439.

56. Suzuki, T.; Kozaki, J.; Sugawara, R.; Mori, K.; Appl. Entomol. Zool. 1984, 19,15 .

57. Banks, H. J.; Fields, P. G. Em Stored Grain Ecosystems; Jayas, D. S.; White, N. D.; Muir, G., eds.; Marcell Dekker: New York, 1995, p. 353-409.

58. http://www.pheroshop.com, acessada em Agosto 2004

59. http://www.biocontrole.com.br, acessada em Agosto 2004.

60. Thomson, D.; Brunner, J.; Gut, L.; Judd, G.; Knight, A.; IOBC wprs Bulletin 2001, 24, 23.

61. Charmillot, P-J.; Hofer, D.; Pasquier, D.; Entomol. Exp. Appl. 2001, 94, 211. 